\title{
Los primeros 100 manuscritos de médicos del Hospital Ángeles León
}

\author{
Luis Gerardo Domínguez-Carrillo, ${ }^{1}$ Javier Guillermo Domínguez-Herrera, ${ }^{2}$ Ernesto Marín-Santillán ${ }^{3}$
}

En marzo de 2014 se constituyó el Comité de Publicaciones Médicas del Hospital Ángeles León (HAL) con la misión de involucrar a los médicos de la institución en la elaboración de manuscritos con el fin de publicarlos. Cumpliendo así uno de los objetivos primordiales que debe alcanzar el profesionista de la medicina, que es transmitir sus experiencias, siendo la publicación una manera de lograrlo. En un reporte previo se dieron a conocer las características del comité, sus lineamientos y los resultados obtenidos a un año de trabajo. En esta ocasión, a tres años de su formación, presentamos las experiencias logradas durante la elaboración de los primeros 100 manuscritos de médicos del HAL, así como sus características y evolución.

Se revisaron los primeros 100 manuscritos realizados por médicos del HAL clasificándolos por: a) su origen de división (medicina, cirugía, ginecología-obstetricia y pediatría); b) por la característica del manuscrito (artículo original, de revisión, caso clínico, imagen en medicina, historia de la medicina); c) por su estatus al momento de la redacción de este reporte: publicado, en prensa, en revisión; d) por nombre de la revista a la que se envió el manuscrito; e) por el número de médicos involucrados en la elaboración en relación con la cifra de médicos de la institución y por último f) número de manuscritos que requirieron modificación solicitada por los revisores de las publicaciones

\footnotetext{
${ }^{1}$ Especialista en Medicina de Rehabilitación. Profesor de la Facultad de Medicina de León, Universidad de Guanajuato, México.

2 Médico Internista. Jefe de Enseñanza del Hospital Ángeles León.

${ }^{3}$ Cirujano General. Director Médico del Hospital Ángeles León.
}

Correspondencia:

Acad. Dr. Luis Gerardo Domínguez-Carrillo

Correo electrónico: Igdominguez@hotmail.com

Aceptado: 29-03-2017.

Este artículo puede ser consultado en versión completa en http:// www.medigraphic.com/actamedica respectivas antes de su total aceptación, anotando en las referencias bibliográficas los artículos publicados.

A tres años de la formación del Comité de Publicaciones del HAL, sus acciones reportan asesoría, revisión y corrección de 100 manuscritos que corresponden a la siguiente clasificación: 14 artículos originales, siete de revisión, 39 de casos clínicos, 36 de imágenes en medicina, dos manuscritos de historia de la medicina, una carta al editor y un capítulo para el Libro de Medicina Interna (Figura 1). Los temas quirúrgicos ocuparon $45 \%$ de los manuscritos; los temas médicos 51\%; los trabajos de ginecología y obstetricia así como de pediatría $2 \%$, respectivamente.

Los manuscritos se hicieron llegar a 16 publicaciones científicas (Cuadro 1): 12 nacionales, indexadas en el portal Artemisa, reconocidas por la Academia Nacional de Medicina de México y cuatro revistas extranjeras.

De los 100 manuscritos realizados se encuentran al cierre de este reporte: 70 artículos publicados, 1-70 17 artículos en prensa y 13 manuscritos en revisión por comités editoriales que alcanzan una producción de 2.7 manuscritos/mes durante los tres años de actividad (Figuras 2 y 3). El comité asesoró a 70 médicos (46\%) de la institución en la elaboración de los manuscritos, además de 12 internos de pregrado.

Por parte del comité las acciones de asesoría reportan: haber revisado 100 documentos que incluyeron 743 páginas, 64 cuadros, 147 fotografías, 211 pies de gráfica o figura y 1,312 referencias bibliográficas. Se efectuaron 150 entrevistas personales; 745 correos electrónicos; 243 Ilamadas telefónicas; apoyo en 39 modificaciones a manuscritos solicitadas por comités editoriales; presentación de 36 informes mensuales de las acciones del comité así como tres informes anuales y cinco exposiciones de resultados en sesión general de la institución, dos de ellas forman parte de las sesiones del Capítulo Centro de la Academia Nacional de Medicina.

\section{DISCUSIÓN}

Los profesionales de la medicina publican para compartir con la comunidad médica sus conocimientos y experien- 
cias. Las prerrogativas de esta información se clasifican en diferentes rubros que implican que dicha información debe: a) enriquecer el conocimiento y comprensión de una determinada enfermedad; b) presentar resultados de estudios realizados; c) comunicar nuevos métodos de diagnóstico o tratamiento; d) reflexionar sobre los resultados publicados; e) revisar un tema específico; f) desarrollar un caso clínico; g) mostrar una imagen ilustrativa y h) externar las ideas y opiniones en ensayos literarios sobre un tema específico relacionado con la medicina.

Figura 1. 100 manuscritos del Hospital Ángeles León.

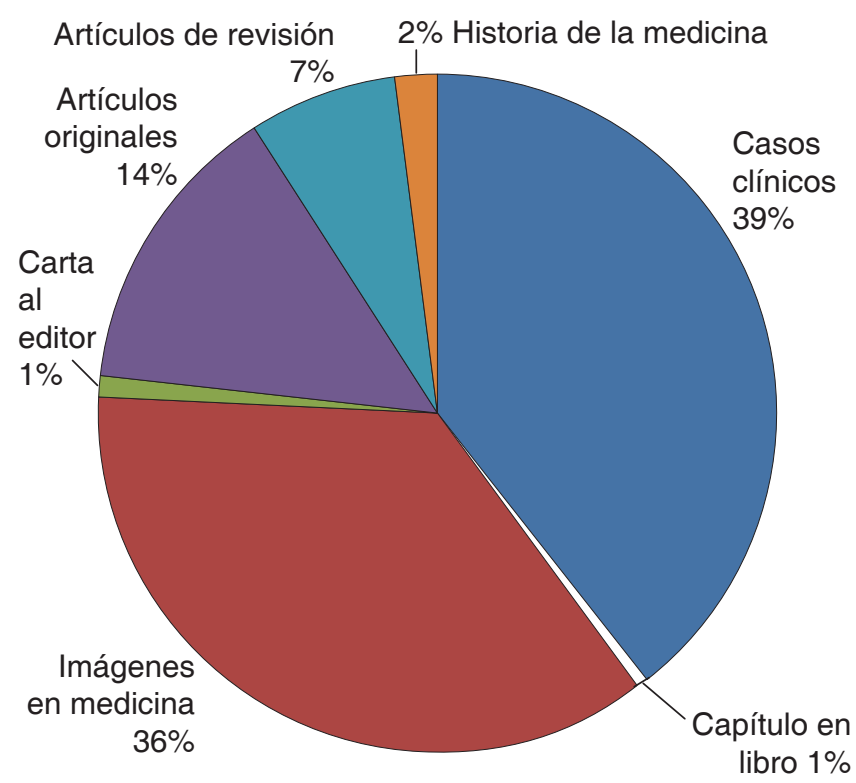

La mayoría de los médicos con frecuencia exponemos nuestras experiencias clínicas a través de conferencias o participando en sesiones clínicas. El reto surge cuando se nos solicita expresar esas experiencias por escrito, ya que escribir bien un artículo científico requiere tiempo, estudio y dedicación.

Pese a que existen múltiples revistas médicas en nuestro país, el número de artículos publicados resulta muy esca-

Cuadro 1. Lista de publicaciones científicas en las que aparecen los 70 artículos de médicos del Hospital Ángeles León, de marzo de 2014 a febrero de 2017.

1. Revista Acta Médica Grupo Ángeles

2. Revista de la Facultad de Medicina de la UNAM

3. Revista Cirugía y Cirujanos

4. Revista Atención Familiar UNAM

5. Revista Anestesia de México

6. Revista Ginecología y Obstetricia de México

7. Revista Mexicana de Medicina Física y Rehabilitación

8. Revista Mexicana de Cardiología

9. Revista MD

10. Revista Gastroenterología de México

11. Revista Endoscopia

12. Revista Acta Ortopédica Mexicana

13. Revista Obesity Surgery

14. International Journal of Critical Care and Emergency Medicine

15. Global Journal of Medical and Clinical Case Reports

16. Global Journal Obesity, Diabetes and Metabolic Syndrome

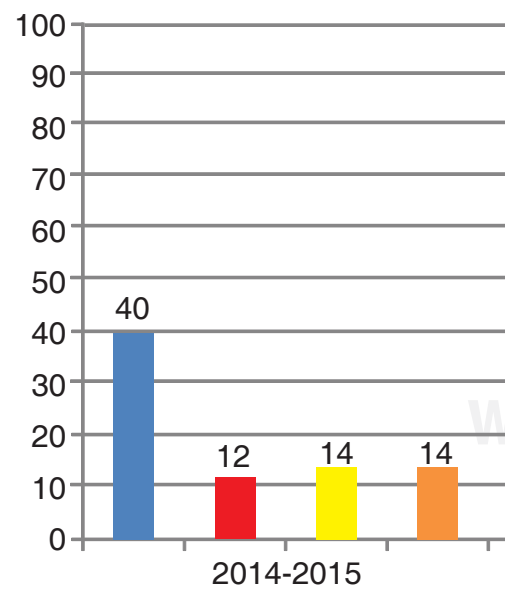

Manuscritos elaborados

Artículos en prensa
Artículos publicados

Manuscritos en revisión
Figura 2.

100 manuscritos del Hospital Ángeles León 20142017. 
Figura 3. 100 manuscritos del HAL 2014-2017.

Promedio 2.7/mes



so en relación con el número de médicos existentes. Los médicos no estamos acostumbrados a escribir, en nuestro medio no existe una cultura para publicar y por lo tanto, escribir un artículo con este fin resulta una tarea compleja.

Existen ciertos atavismos ante una hoja de papel en blanco a la hora de plasmar las ideas por escrito, uno de ellos es asumir que escribir bien es una habilidad innata, cuando en realidad es una cuestión de práctica. Otra limitante es creer que los únicos que lo hacen son los investigadores y expertos reconocidos; sin embargo, todo médico puede llegar a escribir para publicar, incluso los estudiantes de pregrado. Una tercera limitante es pensar que la única forma de lograrlo es enviar trabajos de investigación complejos, situación que también resulta falsa, pues puede escribirse sobre: casos interesantes de la práctica clínica, un signo o una imagen, experiencias personales, análisis críticos constructivos de problemas médicos, reseñas históricas y comentarios sobre estudios incluidos en las revistas, modificaciones a técnicas quirúrgicas, complicaciones y la manera de resolverlas, asociación entre enfermedades, respuestas a un fármaco, abordaje de problemas, epidemiología en nuestro medio de un problema específico en comparación con lo ya publicado, etc. Con respecto al médico que se inicia en la elaboración de un manuscrito con la intención de publicarlo, la presentación de un caso clínico siempre es un buen comienzo; no obstante, no todas las revistas médicas aceptan casos clínicos, pues la mayoría de los comités editoriales de publicaciones científicas coinciden en que la comunicación de casos clínicos contribuye poco al conocimiento, arguyendo que contiene un nivel bajo de evidencia científica; sin embargo, su objetivo es exponer aspectos nuevos o instructivos de una enfermedad determinada, siendo el informe riguroso de casos clínicos el primer paso en la investigación clínica, además de ser una forma de comunicación que constituye una técnica fundamental en la enseñanza médica que permite recordar mensajes, favorece la comprensión de una guía práctica clínica, estimula el razonamiento y el manejo de la incertidumbre, aparte de incrementar las posibilidades de diagnóstico diferencial o motivar la búsqueda de mayor información.

La importancia de la información escrita radica en su trascendencia y gracias a la comunicación electrónica que está prácticamente al alcance de todos los interesados, como lo demuestra la información proporcionada sobre el número de artículos visitados en la Revista Acta Médica Grupo Ángeles, que de enero a septiembre de 2016 reporta la cifra de 662,448 PDF consultados en la red, con un promedio/día de 2,400 visitas abarcando 70 países del orbe, quizá limitada en su difusión por el idioma, pues únicamente los hispanoparlantes pueden acceder a la totalidad de los artículos, mientras que el resto sólo tiene acceso al resumen en idioma inglés o a una traducción, no de la mejor calidad, a través de traductores automatizados.

Consideramos que la formación del comité de publicaciones del Hospital Ángeles León ha sido fructífera, la meta inicial de ocho manuscritos/año ha sido ampliamente superada con un promedio de 33 manuscritos/año. Se logró involucrar (por convicción y no por obligación) a prácticamente $50 \%$ de los médicos de la institución en la realización de artículos, con una producción de 2.7 ma- 
nuscritos/mes de manera constante a lo largo de tres años, contando con $70 \%$ de ellos ya editados. Los 17 artículos en prensa permitirán dar a conocer a los médicos de la institución en al menos 10 diferentes revistas durante 2017. Por otra parte, se cuenta con 13 manuscritos elaborados que ya están en valoración por comités editoriales, que de ser aceptados incrementarán las posibilidades de cubrir parte de 2018. En cuanto a la información vertida en los artículos publicados estamos satisfechos, ya que las revistas científicas en las que han aparecido cuentan con comités editoriales que evalúan la calidad de los manuscritos, al ser examinados por pares los documentos enviados se han sometido a revisión por expertos en el tema que emiten un juicio tanto de la calidad de los datos como de la veracidad de las conclusiones. Las modificaciones a 39 manuscritos enviados han sido siempre atinadas, mejorando la calidad y contenido de los documentos. Sirvan estas líneas para agradecer a los revisores su dedicación profesional, la cual resulta esencial para obtener un producto de utilidad para la comunidad médica interesada en el tema respectivo de cada documento.

En cuanto a las perspectivas a futuro de este comité se ha establecido una relación estrecha con el comité de investigación, iniciando con una "lluvia de ideas" de todas las divisiones y departamentos de la institución para valorar propuestas de investigación factibles, así como la redacción de los protocolos respectivos con la intención de promover un mayor número de artículos originales, al tiempo de tratar de mantener los números alcanzados en cuanto a la producción de manuscritos. La tarea es un reto que requiere incrementar el esfuerzo y la conjunción multidisciplinaria en un ambiente cordial que ha sido la característica primordial de este comité.

Para concluir, este comité considera que el médico que escribe y publica es un mejor médico, al menos en el tópico que desarrolla en su manuscrito, pues debe llevar a cabo una verdadera actualización del tema al revisar la bibliografía relacionada con su artículo, amén de trascender al dejar algo por escrito y cumplir (como lo establece el Dr. Ruy Pérez Tamayo) con las tres obligaciones que todo médico debe desempeñar en la medida de sus posibilidades: "cuidar la salud y combatir la enfermedad, compartir sus conocimientos con todo aquel que pueda beneficiarse de ellos (qué mejor que a través de la publicación) y contribuir a enriquecerlos por medio de la investigación médica".

\section{REFERENCIAS}

1. Sandoval LC. Vía aérea difícil: implicaciones en anestesia. Primera de dos partes. Anestesia en México. 2014; 26 (1): 18-34.

2. Sandoval LC. Vía aérea difícil: implicaciones en anestesia. Segunda de dos partes. Anestesia en México. 2014; 26 (3): 18-30.

3. Mora CJ, Alcocer MJ, Domínguez CL. Plasmocitoma óseo solitario. Acta Med Gpo Ang. 2014; 12: 150-151.
4. Sandoval LC, López AL, Hasslacher AJ, Mora CJ, Domínguez CL. Lesión del cono medular por anestesia espinal: Un diagnóstico clínico-radiológico. Acta Med Gpo Ang. 2014; 12: 141-145.

5. Arceo-Navarro A, Harrison-Gómez C, Sánchez-Lezama F, Armenta-Flores R, Domínguez-Carrillo LG. Arteria descendente anterior en síndrome de Wellens: cuatro perspectivas. Acta Med. 2014; 12 (3): 154-155.

6. Marín SE, Domínguez CL. Artículos científicos en el Hospital Ángeles León. Acta Médica. 2014; 12 (3): 156-159.

7. Marín SE, López FS, Domínguez CL. Indicadores quirúrgicos en el HAL. Acta Med Gpo Ang. 2014; 12: 160-163.

8. Mora CJ, Baca PE, Harrison GC, Sánchez LF et al. Hepatocarcinoma manifestado como dolor torácico. Acta Med Gpo Ang. 2014; 12: 203-206.

9. Alcocer MJ, Güemes E, Mora CJ. Síndrome de Maffucci. Acta Med Gpo Ang. 2014; 12: 211-214.

10. Domínguez-Gasca LG, Mora-Constantino J, Domínguez-Carrillo LG. Quiste aneurismático. Acta Med Gpo Ang. 2014; 12 (4): 217-218.

11. Mora CJ, Arellano AG, Domínguez CL. Neoplasia endocrina múltiple: síndrome de Werner. 2014; 12: 221-222.

12. Baca PE, Peralta A, Argüello BJ, Castañeda OA, Hernández AM, Ochoa GP. Tratamiento laparoscópico del quiste de colédoco en niños. Acta Med Gpo Ang. 2015; 13: 38-41.

13. Domínguez-Gasca LG, Alcocer-Maldonado JL, Domínguez-Carrillo LG. Hernia discal masiva: itratamiento quirúrgico o conservador? Acta Med Gpo Ang. 2015; 13: 55-56.

14. Arellano AG, Domínguez CL. Síndrome de Hoffman. Acta Med Gpo Ang. 2015; 13: 26-29.

15. Domínguez CL, Alcocer MJ, Mora CJ, Domínguez CL. Compresión radicular por quiste sinovial facetario. Acta Med Gpo Ang. 2015; 13: 34-37.

16. Ramírez AC, Domínguez CL, Mora CJ. Tumor de Warthin. Acta Med Gpo Ang. 2015; 13: 42-45.

17. Domínguez GL, Mora CJ, Gómez VA, Domínguez CL. Comparación del ángulo de Ferguson en bipedestación y decúbito. Acta Med Gpo Ang. 2015; 13: 82-85.

18. Hasslacher AJ, Arellano AG, Mora CJ. Quiste de Tarlov: un reto diagnóstico y de manejo. Acta Med Gpo Ang. 2015; 13: 104-108.

19. Alcocer MJ. Domínguez GL. Granuloma eosinófilo de la tercera vértebra cervical. Acta Med Gpo Ang. 2015; 13: 124-125.

20. García LA, Nez EH, López BF, López FS, Padilla SM, Domínguez $\mathrm{CL}$. Indicadores de la $\mathrm{UCl}$ del Hospital Ángeles León en pacientes postquirúrgicos. Acta Med Gpo Ang. 2015; 13: 137-143.

21. Ramírez AC, Domínguez CL. Síndrome de Frey. Acta Med Gpo Ang. 2015; 13: 177-179.

22. Arellano AG, Baca PE, Aguirre TJ. Diarrea en viajero frecuente. Acta Med Gpo Ang. 2015; 13: 190-193.

23. Tinoco TL, García LA, Marín SE. Lipoblastoma en paciente adulto: presentación inusual. Acta Med Gpo Ang. 2015; 13: 180-183.

24. Domínguez CL, Marín SE, Domínguez HJ. Comité de publicaciones del Hospital Ángeles León: un año de experiencia. Acta Med Gpo Ang. 2015; 13: 196-198.

25. Alcocer MJ. El cerebro en el libro "De Humani Corporis Fabrica" de Vesalio. Acta Med Gpo Ang. 2015; 13: 199-205.

26. Domínguez CL. Síndrome de Burnout. Acta Med Gpo Ang. 2015; 13: 208.

27. Arellano AG, Aguirre TJ. Gastroenteritis eosinofílica. Revisión a propósito de un caso. Acta Med Gpo Ang. 2015; 13: 238-243.

28. Alcocer MJ, Mora CJ, Domínguez CL. Hemangioblastoma supratentorial. Acta Med Gpo Ang. 2015; 13: 255-258.

29. Alcocer MJ. Glioblastoma multiforme con metástasis leptomeníngea espinal. Acta Med Gpo Ang. 2015; 13: 275-276.

30. Arellano AG, Salas Gl, López BA, Domínguez CL. Monitoreo Holter prolongado en pacientes con ictus establecido. Acta Med Gpo Ang. 2016; 14: 32-35.

31. Mora CJ, Aguirre TJ, Domínguez CL. Adamantinoma. Acta Med Gpo Ang. 2016; 14: 39-40. 
32. Arellano AG, Domínguez CL. Lesión del nervio espinal izquierdo por vaciamiento ganglionar cervical. Acta Med Gpo Ang. 2016; 14:104-107.

33. Domínguez HJ, Sandoval LC. Prevalencia del dolor postquirúrgico en el Hospital Ángeles León. Acta Med Gpo Ang. 2016; 14: 84-89.

34. González PF. Domínguez CL. Contractura del platisma secundaria a parálisis facial por herpes zoster. Acta Med Gpo Ang. 2016; 14: 119-120.

35. Arellano AG, Magaña RJ. Síndrome de Sjögren. Acta Med Gpo Ang. 2016; 14: 90-100.

36. Mora CJ, Castilla BJ, Bribiesca JC. Divertículo esofágico bilateral de Killian-Jamieson. Acta Med Gpo Ang. 2016; 14: 117-118.

37. Tinoco TL, Gálvez VR, Marín SE. Pseudomixoma peritoneal manejado con citorreducción e HIPEC. Acta Med Gpo Ang. 2016; 14: 165-170.

38. Arellano AG, López BA, García LA, Nez EV, Castilla BJ. Bacteriemia por Cedecea lapagei: un caso en México. Acta Med Gpo Ang. 2016; 14: $176-178$

39. Hasslacher AJ, Mora CJ. Síndrome de lesión medular del cordón posterior. Acta Med Gpo Ang. 2016; 14: 179-180.

40. García LA, Nez EV, Magaña RJ, Domínguez CL. Empiema necessitatis. Acta Med Gpo Ang 2016; 14: 181.

41. Tinoco TL, Aguirre TJ, Domínguez CL. Sarcoma fibromixoide. Acta Med Gpo Ang. 2016; 14: 230-234.

42. Alcocer MJ, Domínguez GL, Mora CJ, Domínguez CL. Inestabilidad atlantoaxoidea: a propósito de un caso. Acta Med Gpo Ang. 2016; 14: 235-239.

43. García LA, Nez EV, Domínguez CL. Síndrome de Eagle. Acta Med Gpo Ang. 2016:14: 244-247.

44. Alcocer MJ. Lesión Grado IV del ligamento nucal. Acta Med Gpo Ang. 2016; 14: 25i-252

45. Suarez NJ, Mason CT, Juárez J, Castilla BJ. Divertículo de Meckel. Acta Med Gpo Ang. 2016; 14: 253-254.

46. Arceo NA, Harrison GC, Sánchez LF, Armenta FR. Síndrome de Wellens. Rev Fac Med UNAM. 2014; 57: 38-39.

47. Domínguez GL, Mora CJ, Domínguez CL. Miositis osificante. Rev Fac Med UNAM. 2016; 59: 19-20.

48. Gálvez-Valdovinos R, Cruz-Vigo JL, Marín-Santillán E, FunesRodríguez JF, López-Ambriz G, Domínguez-Carrillo LG. Cardiopexy with teres ligament in patients with hiatal hernia and previous sleeve gastrectomy: an alternative solution for gastroesophageal reflux disease. Obes Surg. 2015; 25: 1539-1543.

49. Gálvez VR, Hernández LR, López AG, Ramme CC. Dispositivo intrauterino traslocado con ectasia ureteropielocalicial. Ginecol Obstet Mex. 2015; 83: 635-640.

50. Domínguez GL, Alcocer MJ, Mora CJ. Compresión del ganglio radicular por quiste sinovial facetario: reporte de dos casos. Rev Mex Med Fis Rehab. 2015; 27: 24-27.

51. Domínguez CL, Alcocer MJ, Domínguez GL. Hernia discal masiva: Rehabilitación. Rev Mex Med Fis Rehab. 2015; 27: 86-93.
52. Arceo NA, Harrison GC, Sánchez LF, Arredondo AV. Effusive-constrictive pericarditis: a review. Rev Mex Cardiol. 2015; 26: 140-148.

53. Sánchez LF, Harrison GC, Arceo NA, Arredondo AV, Armenta FR, Domínguez CL. Mean pulmonary pressure estimation by echocardiography: three equations comparison. Rev Mex Cardiol. 2015; 26: 108-112.

54. Sánchez LF, Harrison GC, Arceo NA, Arredondo AV, Domínguez CL. Echocardiographic findings in 1,468 cases. Rev Mex Cardiol. 2016; 27: 250-255.

55. Gálvez VR, Marín SE, Funes RJ. Manga gástrica laparoscópica en vólvulo gástrico secundario a eventración diafragmática en paciente adulto. Cir Cir. 2016; 84: 140-143.

56. Hasslacher AJ, Arellano AG, Funes RJ, López FS, Torres ZF, Domínguez $\mathrm{CL}$. Derivación ventrículo-vesicular: una alternativa en el tratamiento de hidrocefalia. Cir Cir. 2016; 84: 225-229.

57. González AM, Linares SB, Ramírez BE, Salcedo GJ, Flores $\mathrm{CH}$. Indicaciones para esofagogastroduodenoscopia. Utilidad de las guías de la Sociedad Americana de Endoscopia Gastrointestinal (ASGE) en un hospital del bajío mexicano. Endoscopia. 2015; 27: 156-157.

58. Gálvez VR. Ectopia renal cruzada: riñón discoide o en "torta". Rev MD. 2016; 7: 111.

59. Tinoco TL, Aguirre TJ. Encondroma protuberans de pared torácica. Rev MD. 2016; 8: 33 .

60. Magaña RJ, Domínguez CL. Sarcoma sinovial intraabdominal. Rev Gastroenterol Mex. 2016; 81: 223-224.

61. Gálvez VR, Arellano AG, Gutiérrez VJ, Domínguez GL. Pasteurella canis as a cause of septic arthritis and soft tissue infection after sheep bite: a case report. GJMCR. 2016; 3: 12-14.

62. Gálvez VR, Marín SE, López AG. Efficacy of early laparoscopic surgery in acute abdomen secondary to perforated jejunal diverticulitis. Int J Crit Care Emerg Med. 2016; 2: 20-22.

63. Domínguez GL, Magaña RJ. Fractura del piramidal. Rev Aten Fam. 2017; 24: 47-48.

64. Magaña RJ, Domínguez GL, García LA. Lesión del músculo ilíaco por ejercicio inadecuado. Rev Acta Ortop Mex. 2016; 30: 154-157.

65. Baca PE. Domínguez CL. Hernia gigante inguino-escrotal. Rev Gastroent Mex. 2017; 82: 89-91.

66. Gálvez VR. Complications from laparoscopic adjustable gastric banding: 364 cases experience. Global J Obes Diabetes Metb Synd. 2017; 4: 1-4.

67. García LA, Nez HV, López BF, Mora CJ. Granulomatosis con poliangeitis diagnosticada en UCI. Acta Med. 2017; 15: 47-51.

68. Tinoco TL, Marín SE, Murguía PM, Domínguez CL. Tumor sólido pseudopailar de páncreas. Acta Med. 2017; 15: 52-56.

69. Magaña RJ, Domínguez GL, Domínguez CL. Compresión radicular L5 extraforaminal por músculo psoas accesorio. Acta Med. 2017; 15:66-67.

70. Arellano AG, Gálvez VR, López AG, Magaña RJ, Aguirre TJ, Pliego RB, Núñez ME. Síndrome de Conn. Acta Med. 2017; 15: 68-69. 